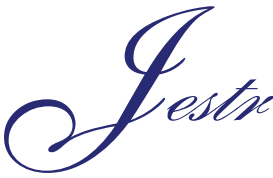

Journal of Engineering Science and Technology Review 3 (1) (2010) 128-135

Research Article
JOURNAL OF

Engineering Science and

Technology Review

www.jestr.org

\title{
Implementation of Shape - Based Matching Vision System in Flexible Manufacturing System
}

\author{
L. W. Teck, M. Sulaiman, H. N. M. Shah and R. Omar \\ Faculty of Electrical Engineering, Universiti Teknikal Malaysia Melaka, Karung Berkunci 1752, Pejabat Pos Durian Tunggal, 76109. \\ Durian Tunggal, Melaka.
}

Received 16 February 2010; Accepted 25 May 2010

\begin{abstract}
This research is regarding the application of a vision algorithm to monitor the operations of a system in order to control the decision making concerning jobs and work pieces recognition that are to be made during system operation in real time. This paper stress on the vision algorithm used which mainly focus on the shape matching properties of the product. The main focus of this paper is on the development of an adaptive training phase of the vision system, which is the creation of a flexible Region of Interest capability that is able to adapt to various type of applications and purposes depending on the users' requirements. Additionally, an independent stand-alone control scheme was used to enable this system to be used in various types of manufacturing configurations. The system was tested on a number of different images with various characteristics and properties to determine the reliability and accuracy of the system in respect to different conditions and combination of different training traits.
\end{abstract}

Keywords: Flexible Region of Interest, Shape Recognition, Image Processing, Template Matching, FMS Sequence Control.

\section{Introduction}

A flexible manufacturing system is an adaptive and dynamic system that cater to a wide range of different jobs where each involves a set of operations that are required to be done at a predetermine workstation. Flexible and agile manufacturing is of increasing importance in advancing factory automation that keeps a manufacturer in a competitive edge. Flexibility signifies a manufacturing system's ability to adjust to customers' preferences and agility means the system's speed in reconfiguring itself to meet changing demands. Both together make it possible for manufacturer to respond instantly to the market. To achieve a fully flexible automated system, one of the supporting systems is machine vision. Machine Vision is the application of computer vision to industry and manufacturing sectors, mainly focused on machine based image processing. It is also the study of methods and techniques whereby artificial vision system can be constructed and usefully employed in practical applications [1].

\section{Backround}

In most FMS literatures, it can be seen that the current FMS are still not flexible enough and there are a lot of constrains when re-

*E-mail address: omarrosli@yahoo.com

ISSN: 1791-2377 (C) 2010 Kavala Institute of Technology. All rights reserved. searchers attempt to find the most optimize scheduling algorithm in their research. Whether the researchers are trying to improve an existing FMS, simulating or designing a new FMS, the main objective is to find the shortest lead time and higher utilization of facilities. There are a lot of different approach that was studied, scheduling algorithm like taboo search [2], heuristic approach [3], filtered-beam-search [4], branch and bound [5] by using different control theory such as fuzzy logic [6], [7], neural network [4], genetic algorithm [8], [9] and many more. The common point that most of these article share are most likely the set of dispatching rules, including Earliest Due Date, Shortest Processing Time, First Come First Serve, Most Work Remaining, Least Work Remaining, Longest Operation Processing Time and many more. All of these rules are used depending on the type of manufacturing systems and also the objects being manufactured.

Despite many claims that FMS investment should be viewed as a strategic investment in flexibility, the main disadvantage with FMS technologies lies, paradoxically, in its inflexibility. FMS are flexible in that they can, in the short-term, produce a range of known products [10]. However, the complexity necessary to automatically achieve short-term flexibility makes it difficult to introduce new families of products into the system, and certainly much more difficult than in a manual shop. Similarly, when new machines are to be added (or old ones updated) it can be very 
costly. Changes in system configuration require time-consuming, expensive alteration to the software [11], [12] particularly in complex Western Systems.

In the area of Machine Vision inspection system, a lot of different approaches have been studied intensively and plenty of machine vision software are available. B. Mehenni and M.A. Wahab [13] studies on the Automatic Pattern Recognition and Inspection System (APRIS). They divide pattern recognition and inspection problems into two distinct classes, which checks the product for completeness and searching for blemishes and other flaws. They used an ASIC implementation system together with FPGAs based static RAM technology from Xilinx to produce their prototype. Another visual inspection for quality assessment are studied by Piuri V. and Scotti F. [14]. The aim of their research is to develop a defect detection system for melamine laminated particle board. They extract knowledge from the printed matter to guarantee higher defect detection capabilities, the main criteria consider is the texture, color and shape features. Denni Kurniawan and Riza Sulaiman [15] studied the design and implementation of automatic visual inspection system in automatic control system based on Programmable Logic Controller (PLC). The visual inspection system are developed using Visual $\mathrm{C}++6.0$ and Vision SDK 1.2 from Microsoft. The aim of their research is to inspect the size of the bottles of the MAPS bottling system. Another research based on HALCON application for Shape-Based Matching is done by Xuebin Xu, Xinman Zhang, Jiuqiang Han, Cailing Wu [16]. They describe the process involved in a basic shape based matching algorithm and the detail programming language used by HALCON. The basic concept of image matching is shown in Figure. 1.

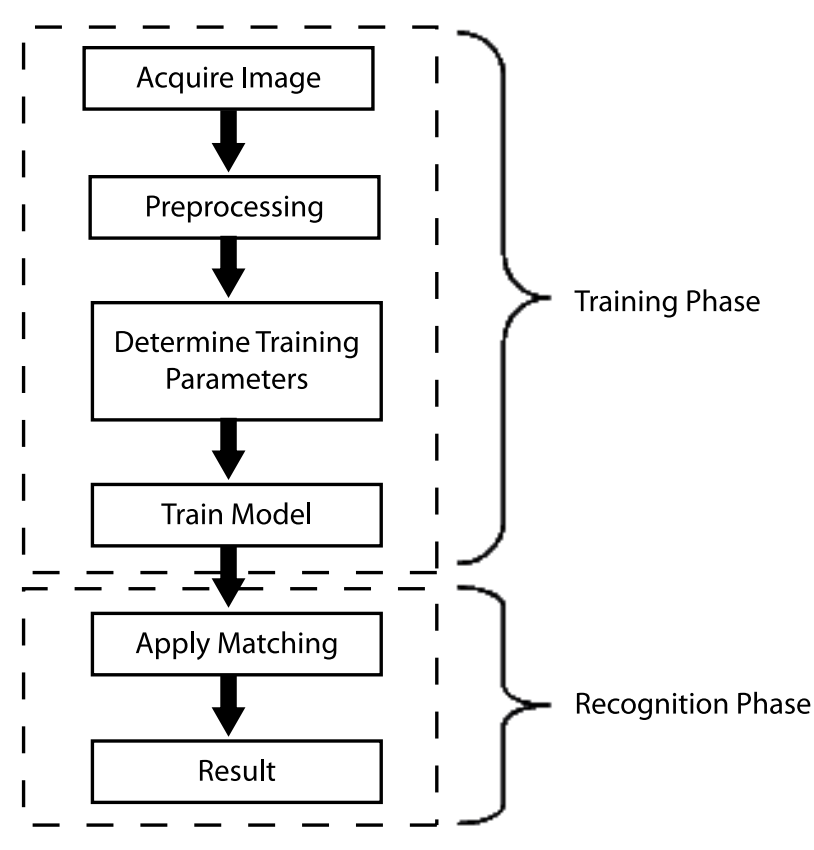

Figure 1. Basic framework for image matching

In all the above research, the machine vision system and software used has two common similarities, first is the three basic framework of the process involved; image acquisition, preprocessing and feature extraction/selection; second is the two phases required for shape matching, the training phase and the recognition phase. In the above research, it seems that most model based vision programs are develop for a specific task and the environment is implicitly coded into the system. Therefore it is difficult to modify the knowledge or extend the scope of such system, and it also requires long development time.

The aim of this research is the design and implementation of a machine vision system for industrial application. The focus of this research will be on shape-based matching, selective Region of Interest and the independent control scheme that can be applied to any flexible manufacturing system, particularly automated visual inspection and vision for automated assembly.

\section{Proposed System}

\section{$\underline{\text { Vision System }}$}

The vision system in this research used is based on HALCON, a machine vision software that provides a comprehensive vision library that we can manipulate into a new system that suits our requirement.

At the training phase, after all the training parameters are determine by the user, an edge detection library are applied to extract the edges of the image, these edges are then saved as a template that will be used for the recognition phase. At the recognition phase, images are then fed to the system to be matched against the template that was created in the training phase. The suggested vision algorithm is as shown in Figure 2.

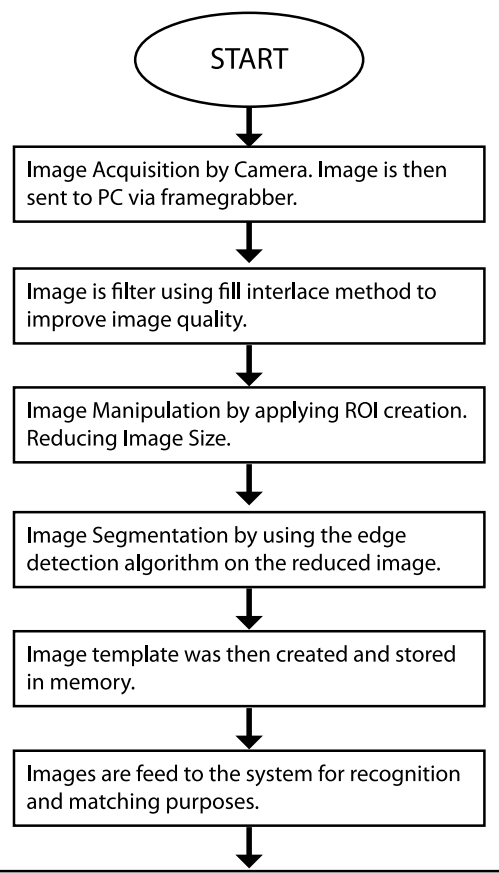

Images will go through the same edge detection algorithm and the reference template will then be applied for matching processes.

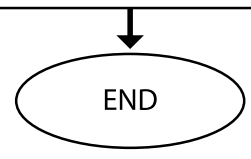

Figure 2. The suggested vision algorithm

As stated above, the aim of this research is the design of the vision algorithm so that it is able to be implemented in a Flexible 
Manufacturing System. Without the pre-knowledge of the objects that are going to be applied, the system is designed to be flexible and smart so that it can be applied without much modification. Therefore the focus will be on the Determine Training Parameters Phase (User define Feature Selection). In order to achieve flexibility and uncertain changes, the main training parameters are determined by the user manually which are located at the user interface.

Feature Selection is a very important part in this system. It not only enables the user to select the critical features, it also minimizes the size of the required matching template therefore saving computation time and operation process. The Feature Selection is done by creating a Region of Interest (ROI), the program is written so that the ROI can be created freehand by using mouse. Furthermore, multiple ROI can be created and combine into one complex ROI. The compelling reason for using multiple ROI is because we are able to extract the object from the background by selecting only the critical criteria that is required to differentiate the wanted object from the background.

The main differences of the suggested vision algorithm with other literatures are the multiple ROI creation in Image Segmentation Step. This user's define ROI creation capability enable users to extract the wanted features by simply using the mouse button, where as in other literature the feature extraction steps are done using combinations of filters and methods to extract the wanted features. Another main advantages of the suggested algorithm is that the system can be easily trained without pre-knowledge of the requirement needed. In all other literature the system was built to solve a specific requirement that was pre-determined, any changes in the requirement will causes the system to be ineffective. The matching criteria of the suggested system are the detection of acceptable features and not the detection of faulty features. The drawback of this algorithm is the reduced accuracy in detecting very fine or very complex images.

\section{$\underline{\text { Control System }}$}

In order to extend the flexibility and adaptability of the system to accommodate different types of FMS configurations, instead of a centralized control scheme, a separate stand-alone control scheme is suggested. In the suggested control scheme, each component of the FMS will act independently, each component (process/cell) will consist of their own machine vision (camera), controller (PC software), and actuator plant (PLC software). This suggested control scheme are able to adapt to system of various flexibility type, where as the dispatching rules utilize are only the First Come First Serve rules. The suggested control scheme is shown in Figure 3, the image on the left shows the single control loop of the suggested system while the image on the right shows the grouping of several control loop together. This control method does not consider the overall system input, instead it considers only the input at its own workstation and gives the output signal for the next process only. Combine with the vision system, the overall control is very simple, for example when Object A is detected Signal A will be produce to turn on Program A, the same goes to Object B, C and so on. The interfaces are used to interact between the Computer and the PLC and also to minimize alterations to the programming at both sides during system modifications.

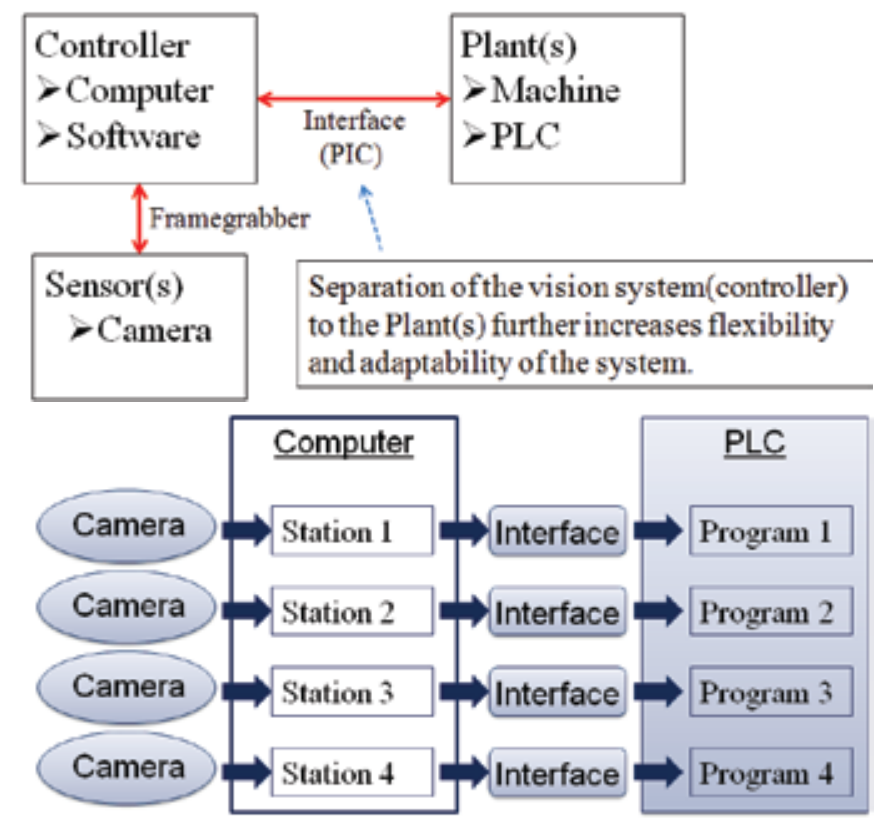

Figure 3. Control Scheme of the suggested system

Similarly, the suggested FMS control scheme is mainly to increase flexibility of the system to be able to adapt in various FMS configurations. In other literatures the focus is always on the scheduling algorithm in order to achieve minimum lead time but the limitations are always bound by a pre-known set of requirements such as process time, process sequence and also workstation configurations type. The suggested FMS control scheme is designed to break down each workstation control into individual parts so that changes can be easily overcome because modifications will only be done at the affected areas and not the overall system. The drawback of this control scheme is the increase in lead time.

\section{Implementation}

The system is then tested by setting it into a system with two pick and place arm and was driven by two conveyor belt as its transportation means, the pick and place system will act as a simple representative of the manufacturing environment, the overall system setup can be seen in Figure 4. On the left is the image of the system used and on the right is the top view representation of the system.

The overall system of all the components including the Controller, Sensor(s), and Plant(s) are connected as stated in Figure 3. The Camera is connected to the Computer via Framegrabber; the PLC is also connected to the Computer via PIC; the Computer will act as both medium and controller in the system. The following steps describe the process cycle of the suggested system:

1. When the object reaches the target location (e.g point 1), the PLC will trigger the feedback signal through the PIC interface to notify the Computer.

2. The Computer will then trigger the Camera to start capture image.

3. When the image was captured, it is then send through Framegrabber into Computer for Image Processing.

4. From Image Processing a serial signal consisting the de- 


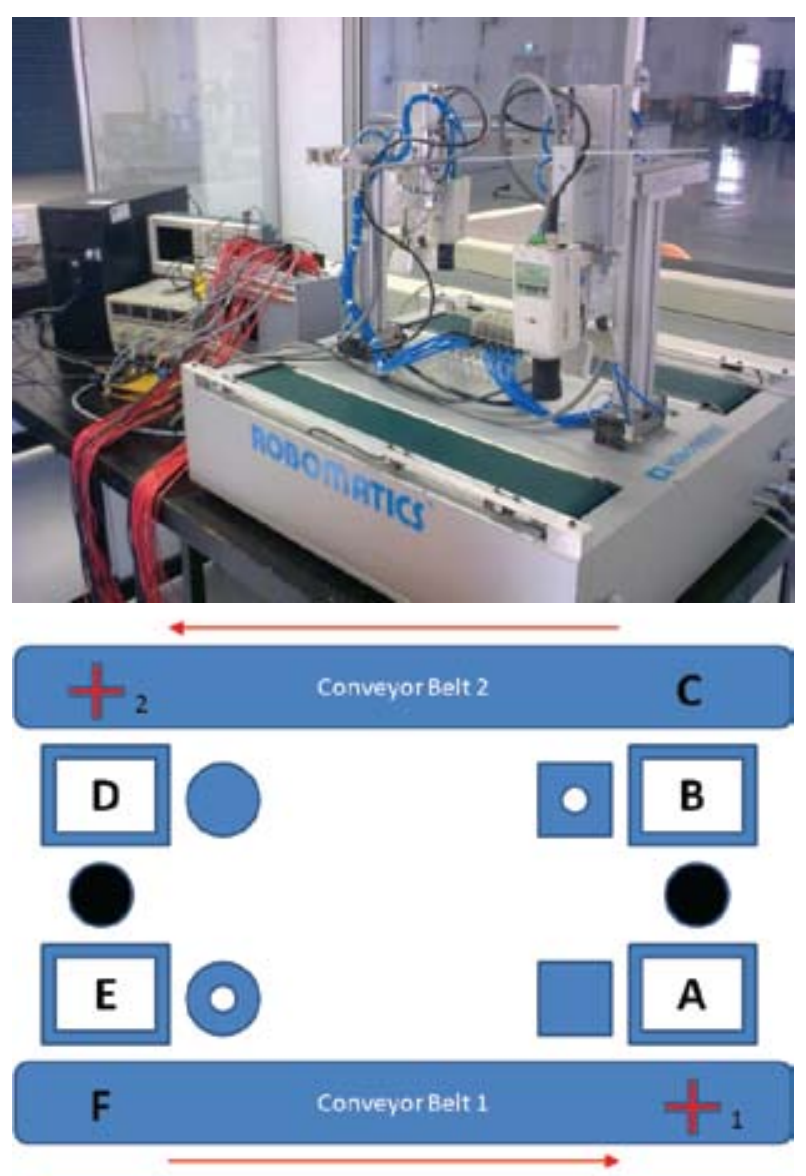

Figure 4. Overall System Setup

cision commands will be sent through PIC interface to PLC.

5. The PLC then will receive the corresponding signal and act accordingly to a pre-determine action.

6. The whole cycle is then repeated when a new object arrives at the target location.

The integration is simple and easy, after the users have determine what action that needs to be done when certain input is detected, just need to connect those output from the system into the PLC. Changes can also be done easily by just exchanging the output wires without the fuss of reprogramming the system. For example, initially the requirement is to locate Object A and Object $\mathrm{B}$ into Location A and Location B while all unrecognized object will be send to the second conveyor belt (assume Location $\mathrm{C}$ ), then the requirement changes into when Object $\mathrm{A}$ and Object $\mathrm{B}$ is found, send it to Location $\mathrm{C}$ and when an unrecognized object is found it is send to Location A. The current wiring connections are output A connected to PLC program A, output B connected to PLC program B and output C connected to PLC program C.

From the given changes, the only modifications needed to be done are to reconnect output $\mathrm{A}$ and output $\mathrm{B}$ to trigger PLC Program $\mathrm{C}$ while output $\mathrm{C}$ to trigger PLC program $\mathrm{A}$. There are a lot more different kinds of combinations that can be done depending on the requirements and also depending on how the users train the vision system. A combination of flexibility of the vision system and of the wiring connection makes this system's adaptability enormous.
As for the vision system, a GUI was made to simplify and allows everyone to be able to use it even without immense knowledge of the vision background. At that GUI page, the procedures for creating a template are very simply, just need to follow the given instruction step by step. Additionally, the buttons have been set to enable and disable accordingly therefore preventing missstep during the template creation.

Referring to Figure 5 shown below, the GUI consists of two image display windows, the top window displays the image during training phase and the bottom window displays the image during matching phase. The image on the left shows the GUI during initializations while the image on the right shows the GUI during matching phase, can be seen that the template (in blue) was created from the reference image at the top window screen. A ROI was drawn to consist of only the required features (in this case the numbers below the barcode), next the image was reduced to show only what the ROI encompass and lastly the template was created after the edge detection algorithm was applied.
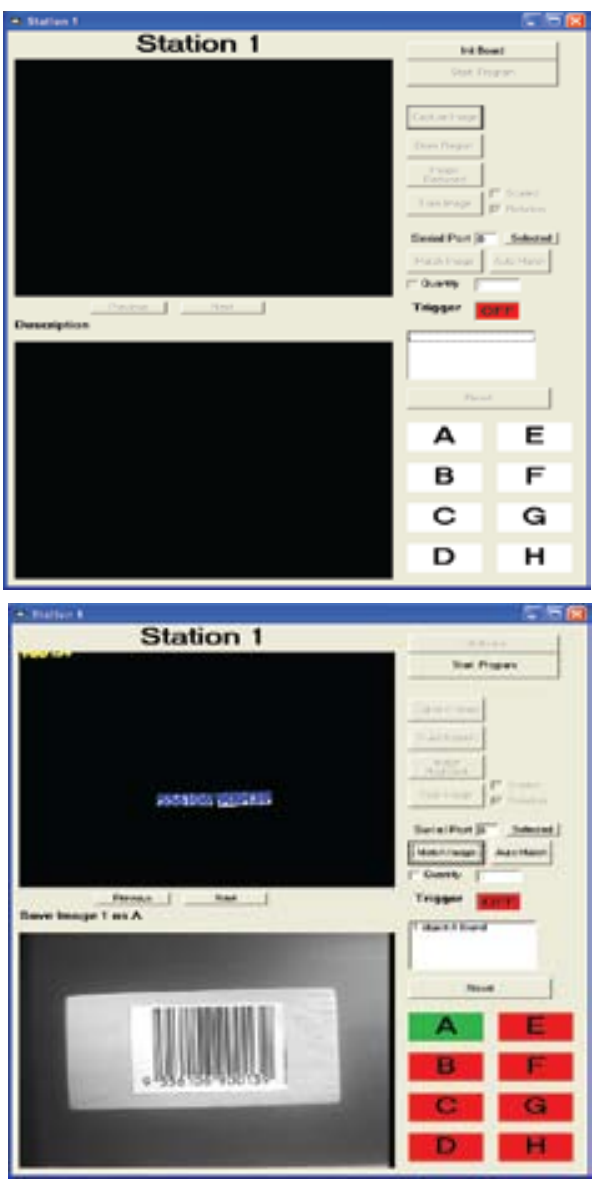

Figure 5. GUI display of the system

Procedures:

Step 1: Start from the button "Init Board", this button turns on the frame grabber in the PC.

Step 2: Click on the "Start Program" button, this activates the camera to capture images continuously. This let users to monitor and calibrate the required setting such as object size (zoom), focus (image clarity), and also brightness (light intensity). 
Step 3: Click on the "Capture Image" button, this captures an image frame after the user has calibrated the camera. This image will be used for the next process.

Step 4: Click on the "Draw Region" button, this button enable users to draw the required ROI using the left mouse button. The ROI always starts and ends at the same point, after the required ROI are drawn click the right mouse button to stop it. It is possible to draw multiple ROI by just clicking the "Draw Region" button again after finishing the previous ROI. When the users are satisfied, then the users can go to the next step.

Step 5: "Image Reduced" button is just to reduce the captured image to a smaller image set by the ROIs drawn.

Step 6: "Train Image" button trains the system to create a template of the reduced image and stores it in the computer memories. Besides this button, there are two tick boxes that trigger additional functions of the system. The "Scaled" tick box, enables the matching to be done with different scale of a same object, the scale is set to be 0.8 to 1.2 of the trained object (1.0). The "Rotation" tick box enables the matching to be done with a difference in angle compared to the angle during training phase, the angle is set to be $-45^{\circ}$ to $+45^{\circ}$ compared to the original train image.

Step 7: "Match Image" button triggers the camera to capture an image to be match with the created template in the computer's memories. When an image is matched, then the GUI will show the output in a text box and at the display below. This button is mainly used for checking whether the trained image is recognizable.

Step 8: "Auto Match" button turns the system into an automatic mode, instead of triggering the camera using the "Match Image" button, the system is triggered by the interface when the triggering conditions are achieved. Click this button once to turn it on and click it again to turn it off, the text display on the button shows the current condition of the system, "Auto Match On" means that the automatic matching system is ON and "Auto Match Off" means that the automatic matching system is OFF. The trigger display below shows when the camera is triggered, during auto match $\mathrm{ON}$ the system will start monitoring the signal sent from the interface. When the triggering conditions have been achieved, the trigger display will show a green color box with the text "ON", which means the camera is being triggered to capture image. Similarly to "Match Image" button, the GUI will show the output in a text box and at the display below.

*the tick box below that shows the text "Quantity" enables the user to check for multiple numbers of required objects. For example when the required object quantity is 2 , the system will only send an output to the interface when 2 of such object is found.

Step 9: Remember to select the correct Serial Port number to assign the correct interface to that Station. The "Selected" button is always enable so that changes in Serial Port can be done easily, but with the restrain that the Serial Port Number selected must exists else there will be an error message.

*the "Reset" button clears all the template stored in the computer's memories

\section{Results}

The potential of the proposed visual algorithm system was the increased flexibility of the program to accommodate changes. At the Feature Selection section, firstly we look at the significance of multiple selective ROI. The system is tested on an image that consists of a combination of objects of different characteristic and requirement.

The first step is to create the ROI, the ROI is drawn by holding the left mouse click while circling the wanted shape. After selecting the ROI, all other image not in the ROI will be removed. The image selected will then go through the vision algorithm for further processing. Major advantages of multiple selective ROI is that the user can decide on the criteria that they required from a combination of different ROI as one object or each ROI as separate objects. An experiment has been done to test the flexibility of the program, a DIP switch has been selected as the main object for this experiment.

\section{Case 1. Checking the Number Of Switches}

In this case, the program is train with the reduce picture of the switch of the DIP Switch. The program will then search for the switch when images are feed to it. The reference image is shown in Figure 6. Figure 6a shows the ROI creation and Figure $6 \mathrm{~b}$ shows the extracted image used to train the system. Start with capturing the image, we then draw the ROI using mouse around the wanted object. The image containing the wanted characteristic will be used as matching template.

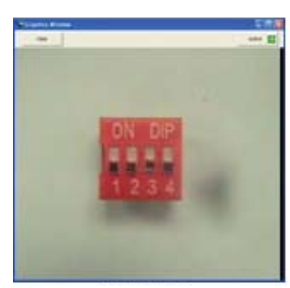

Figure 6

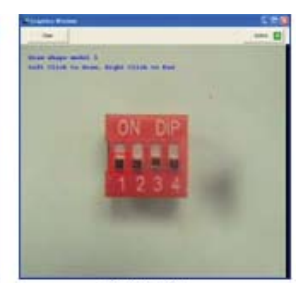

Figure 6a

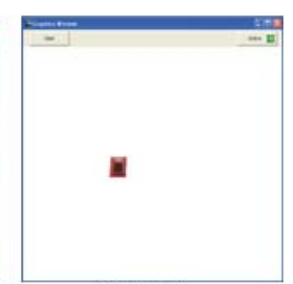

Figure $6 b$
Figure 6. The reference image of a DIP switch used in the experiment Figure 6a. The ROI creation

Figure 6b. The extracted image from the ROI

The matching process is done by using several different switch position configurations and the results are satisfying as long as the object is in the work area. Figure $6 \mathrm{c}, 6 \mathrm{~d}$ and $6 \mathrm{e}$ shows the result when different images are feed to it.

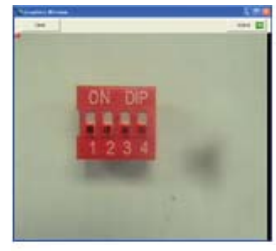

Figure $6 \mathrm{c}$

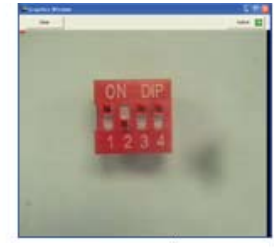

Figure $6 \mathrm{~d}$

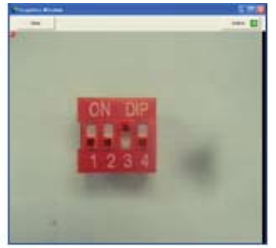

Figure 6e
Figure 6c, 6d \& 6e. Results of the test image

\section{Case 2. Checking the Position Of Switches}

In this case, the program is train to detect the required switch 
configuration, which is switch 1 and 3 must be on, the other two switch 2 and 4 can be in any configurations. The reference image is shown in Figure 7. Figure 7a shows the ROI creation and Figure $7 \mathrm{~b}$ shows the extracted image used to train the system.

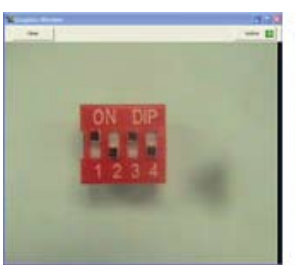

Figure 7

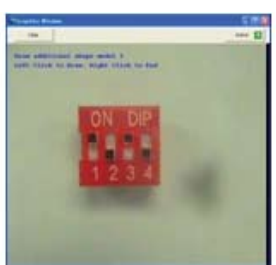

Figure 7a

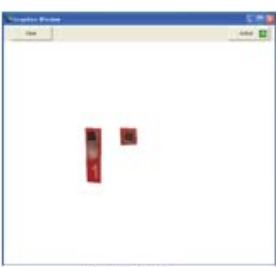

Figure $7 \mathrm{~b}$
Figure 7. The reference image of a DIP switch with switch no 1 and 3 at the on position being used in the experiment

Figure 7a. The ROI creation

Figure 7b. The extracted image used to train the system

The ROI consists of the number 1 with the on position of the switch above it and the on position of the switch 3 . The Number 3 is not included because both small ROI combined into one ROI causing the distance of the two switches to be set still.

Object detection is achievable as long as the position of switch 1 and 3 are at the on position. With no concern on the switch position of the other two switch. Figure 7c, 7d and 7e shows image that satisfied the above conditions while Figure $7 \mathrm{f}$ shows image that does not satisfy the above conditions. Object is no longer detected when the switch position is not at the required position.

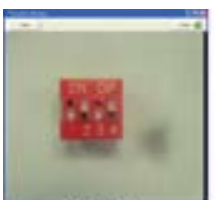

Figure $7 \mathrm{c}$

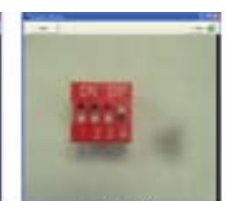

Figure $7 d$

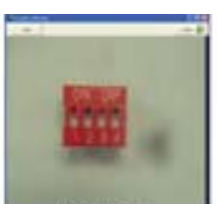

Figure $7 \mathrm{e}$

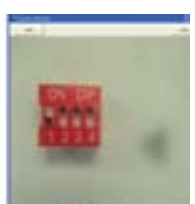

Figure $7 f$
Figure 7c-7f. The images used to test the system and its results

Figure 7c-7e. Images are successfully detected as long as switch 1 and are at the on position

Figure 7f. The image is not detected because the required characteristics are not meet

As for the FMS control scheme, the stand-alone control can be easily adapted to different types of manufacturing configurations since it only affect its own input region and the consequence output action. In this research, two sets of simple pick and place arm are utilized, the control scheme of the system (refer to Figure 4) will consists of two control loop. The first control loop will be Input $\mathrm{A}$ from Camera $\mathrm{A}$ at point 1 triggering output $\mathrm{A}, \mathrm{B}, \mathrm{C}$ and the other control loop will be Input B from Camera B at point 2 triggering output D, E, F.

Additionally, the system can also be used for 3D visual inspection. 3D inspections normally requires either a special camera or a specially built program. By separating the visual inspection into station by station to accommodate the suggested control scheme, a combinations of several cameras located at different angle it is possible to achieve 3D inspection by inspecting two or more $2 \mathrm{D}$ images. By placing another camera at a viewing angle different from the first camera, a 3D inspection technique can be achieved. The experiment sample is shown in Figure 8 while the experiment setup is as shown in Figure 9.

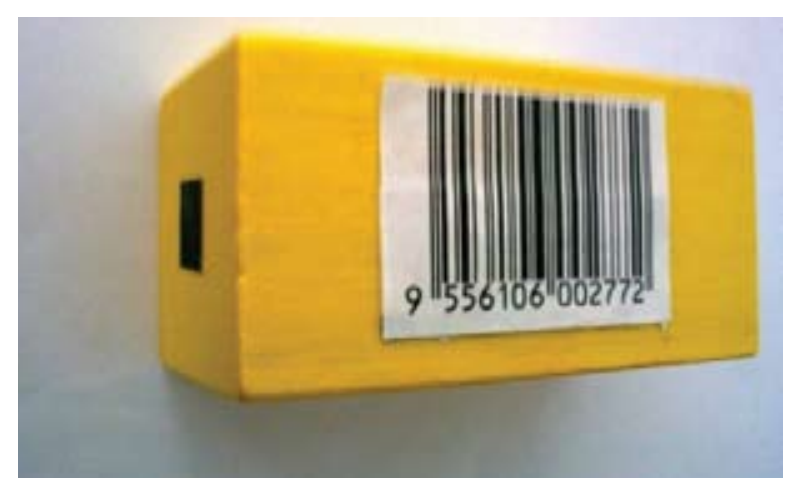

Figure 8. Sample with barcode on its top surface and square shape on its side

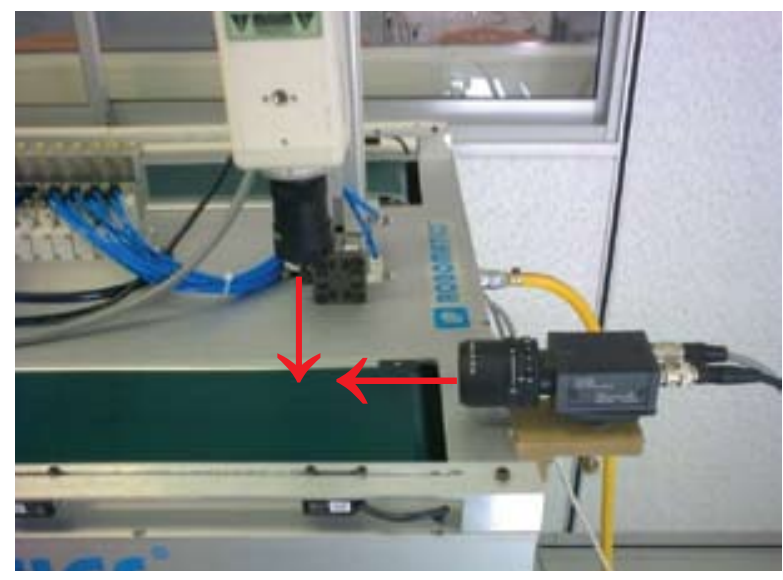

Figure 9. Camera placement for 3D inspection

Two GUI are used in 3D inspection, one for the top and one for the side as shown in Figure 10 and Figure 11 respectively. The

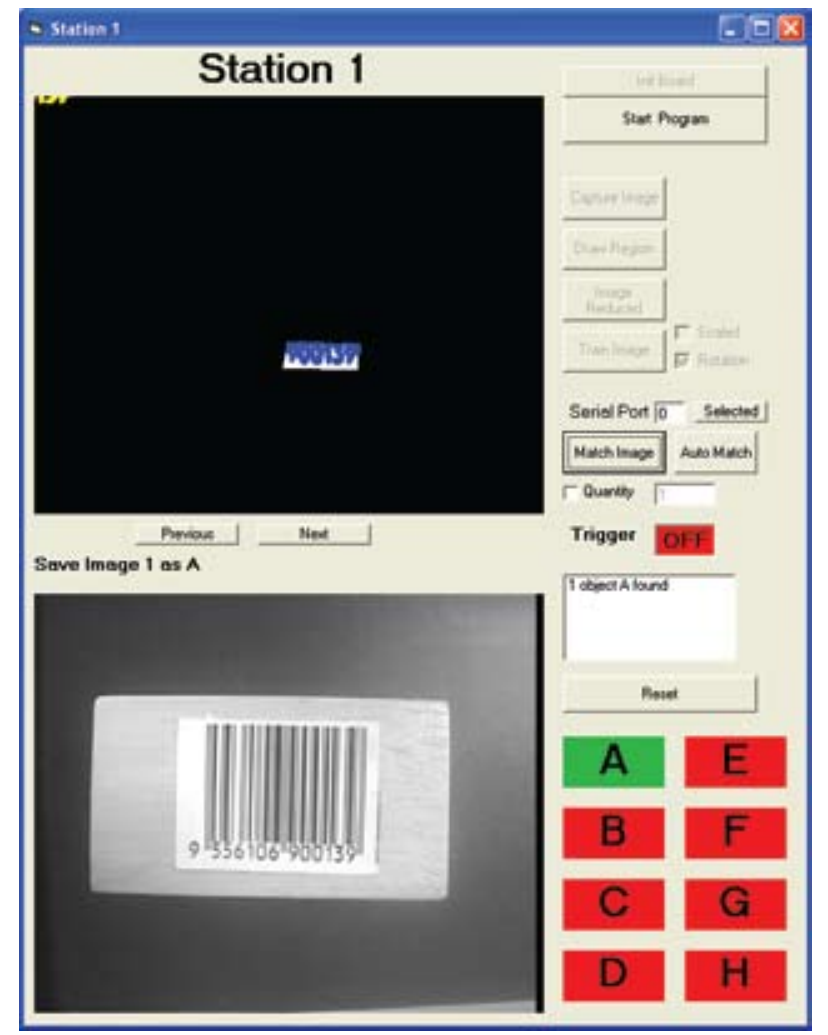

Figure 10. Inspection of top surface 


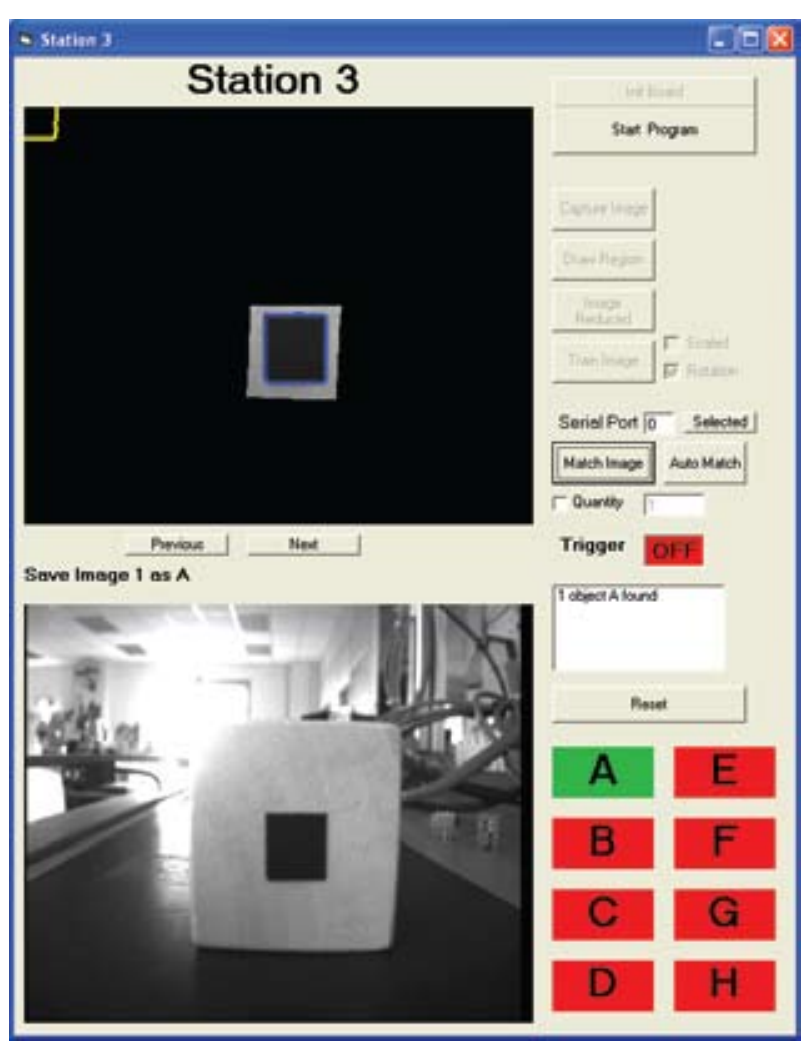

Figure 11. Inspection of side surface

output from both system are then connected in series to the PLC input to duplicate a AND connection since both images must be found in order to be consider an acceptable object.

A few experiments was then implemented with objects of different characteristics, as a results of 100 testing the average correct detections of Acceptable Objects is $92.75 \%$ while the average correct detections of Rejected Objects is $100 \%$, which means the acceptable objects which are rejected is $7.25 \%$ and the rejected objects which are accepted is $0 \%$.

\section{Discussion}

From the results, it can be seen that the system's efficiency is very good, even when the percentage of accept acceptable object is not $100 \%$ but the percentage of reject rejected object is $100 \%$. This is because of the detection scheme that compares only the required features which is the acceptable features detection and not fault detection, therefore the system's efficiency are consider acceptable.

Another advantages of the system is its simplicity and ease of use, since the matching algorithm uses a single edge detection method that was built to process the current environment during training phase instead of predetermined environment setting, the training phase of different objects with different background can be easily achieve, provided the environment setting remains constant from training phase to matching phase. This greatly reduces the time used for setting and tuning of the vision system whenever there are any changes.

\section{Conclusion}

The aim of this paper is to present a shape based matching vision system for Flexible Manufacturing System. In this paper a concept for a flexible ROI creation visual system was presented where the parameters and characteristics can be easily determined by the user. The proposed visual algorithm concept is easily adaptable and extendible, so that this program can be used in most situations as seen fit by the user. This innovative approach allows the user to select and adapt the system according to their requirements. Additionally combine with the stand-alone control concept, this system can be applied to various types of manufacturing configurations. A combinations of its stand alone systems can also be used in 3D visual inspections.

\section{Future Planning}

This project will be continued further by applying the system to another system with a different configuration type. The system will consist of a robotic arm that serves as the transportation means and with three more process stations that is control by PLC. The aim of the project is to show the ease of implementation of the vision system and also to test its reliability and flexibility.

\section{Acknowledgment}

The authors would like to extend their thanks to the Universiti Teknikal Malaysia Melaka (UTeM) for the financial support granted for this research under the Project No: PJP/2008/FKE (6) - S453.

\section{References}

1. Machine Vision: Theory, Algorithms, Practicalities. 3rd Edition by E. R. Davies, (2005).

2. G. Wan, F. Wan, 'Job Shop Scheduling By Taboo Search With Fuzzy Reasoning' Systems, Man and Cybernetics, IEEE International Conference On, 5-8 Oct. 2003, Vol. 2, pp. 1566-1570, (2003).

3. A. Agarwal, S. Colak and E. Eryarsoy, Improvement heuristic for the flow-shop scheduling problem: An adaptive-learning approach. European Journal of Operational Research, Vol. 169(3), pp. 801-815, (2006).
4. Wang Shi-jin, Xi Li Feng, Zhou Bing Hai. Filtered-beam-search based algorithm for dynamic rescheduling in FMS. Robitics and Computer-Integrated Manufacturing, Vol. 23(4), pp. 457-468, (2007).

5. Temel Öncan, Kuban Altinel. 'Exact solution procedures for balanced unidirectional cyclic layout problem'. European Journal of Operational Research, Vol. 189(3), pp. 609-623, (2008).

6. M. A. S. Monfared, S. J. Steiner, 'Fuzzy adaptive scheduling and control systems', Fuzzy Sets and Systems, Vol. 115(2), pp. 231-246, (2000). 
7. H. G. Wang, J. E. Rooda, J. F. Haan, 'Solve Scheduling Problems with a Fuzzy Approach', Fuzzy Syatems, Proceedings of the Fifth IEEE International Conference On, 8-11 Sept. 1996, Vol. 1, pp. 194-198, (1996).

8. F. Pezzella, G. Morganti, G. Ciaschetti, 'A genetic algorithm for the Flexible Job-Shop Scheduling Problem', Computer and Operational Research, Vol. 35(10), pp. 3202-3212, (2008).

9. R. Di Lorenzo, S. Fichera, V. Grasso, 'Scheduling a cellular manufacturing system with GA'. Knowledge-Based Intelligent Electronic Systems, 1998. Proceedings KES '98, Second International Conference On, 21-23 April 1998, Vol. 3, pp. 116-125, (1998).

10. J. Hartley, 'FMS at Work'. pp. 133-151, IFS (Publications) LTD., Bedford, UK, (1984).

11. O. Bjørke, 'Software Production - The Bottleneck of the Future Manufacturing System'. Annals of CIRP, Vol. 4(2), pp. 545-548, (1975).

12. G. K. Hutchinson, A. T. Clementson. 'Manufacturing Control System: An Approach to Reducing Software Costs', Robotics and Computer Integrated Manufacturing, Vol 1(3/4), pp. 271-281, Pergamon, (1984).
13. B. Mehenni, M. A. Wahab, 'APRIS: Automatic Pattern Recognition and Inspection System', Proceedings of IEEE 7th Annual European Computer Conference (COMPEURO'9), (1993).

14. V. Piuri, F. Scotti, M. Roveri, 'Visual Inspection of Particle Boards For Quality Assessment', Image Processing, ICIP 2005. IEEE International Conference, 11-14 Sept 2005, Vol. 3, pp. 521-524, (2005).

15. Denni Kurniawan, Riza Sulaiman: 'Design and Implementation of Visual Inspection System in Automatic Bottling System based on PLC'. Modeling \& Simulation, AICMS 08. 2nd Asia International Conference On 13-25 May 2008. pp. 760-764, (2008).

16. Xuebin Xu, Xinman Zhang, Jiuqiang Han, Cailing Wu: 'HALCON Application for Shape-Based Matching'. Industrial Electronics and Application, ICIEA 2008. 3rd IEEE Conference On 3-5 June 2008. pp. 2431-2434, (2008). 